\title{
Do demand characteristics contribute to minimal ingroup preferences?
}

\author{
Kerry Brew, Taylar Clark, Jordan Feingold-Link, and Hilary Barth \\ Wesleyan University
}

Accepted for publication, Journal of Experimental Child Psychology

This copy: Preprint 2.0 (December 2020)

Word Count: Abstract $=147$ words, Text + References $=8169$ words

\section{Corresponding Author:}

Hilary Barth, Ph.D.

Department of Psychology, Judd Hall

Wesleyan University

207 High St

Middletown, CT 06459

hbarth@wesleyan.edu

Data Availability Statement: Deidentified data will be shared publicly at https://osf.io/925u7/.

Conflict of interest statement: The authors report no conflict of interest.

Acknowledgments: We thank the participants and their families; the Connecticut Science Center; lab coordinators Katherine Williams and Alexandra Zax; research assistants Ilana Ladis, Sarah Corner, Sofia Kinney, Amanda Fiorentino, Maya Layne, Katie Vasquez, Jenny Chelmow, Esha Bhandari, Sarah Aduke Ohiomah, Lauren Barragan, Sophie Charles; and the Wesleyan University Psychology Department. We also thank Dr. Yarrow Dunham, Dr. Chenmu (Julia) Xing, Dr. Royette Dubar, Dr. Andrea Patalano, and Dr. Valerie Nazzaro and the Wesleyan University Quantitative Analysis Center for their contributions and advice. Some of this research was conducted by Kerry Brew in partial completion of the requirements for the M.A. degree in Psychology. This work benefited from Wesleyan University's Ronald E. McNair Post-Baccalaureate Program and NSF DRL-1561214. 


\begin{abstract}
"Minimal group" paradigms investigate social preferences arising from mere group membership. We asked whether demand characteristics contribute to children's apparent minimal group bias in a preregistered experiment $(N=160)$. In a "group" condition, we attempted to replicate findings of bias following assignment to minimal groups. A second closely-matched "no-group" condition retained potential demand characteristics while removing group assignment. Parallel bias in the no-group condition would suggest that demand characteristics contribute to findings of apparent ingroup bias. Three main findings emerged. First, in the group condition, ingroup preference emerged in one of three bias measures only. Second, this preference emerged although participants evaluated ingroup/outgroup photos varying in race/ethnicity between trials. Third, the measure that yielded ingroup preferences in the group condition produced no parallel bias in the no-group condition, consistent with the view that mere membership in a group, not experimental demand, leads to minimal ingroup preferences.
\end{abstract}


Do demand characteristics contribute to minimal ingroup preferences? People often prefer their ingroups, both familiar real-world groups and arbitrary groups assigned by experimenters for the course of a study (Dunham, 2018; Mullen, Brown, \& Smith, 1992; Tajfel, Billig, Bundy, \& Flament, 1971). The "minimal group paradigm" is commonly used to investigate downstream effects of group membership. In the barest forms of the minimal group paradigm, participants are randomly assigned to arbitrary, novel social groups with essentially no meaning in hopes of revealing what follows from the mere fact of group membership (Dunham, 2018). Preferences for the ingroup following minimal group assignment are widely reported.

Minimal group studies of young children have become especially prominent, showing that we can possess minimal ingroup preferences before we have had extensive experience with real-world groups. Children show minimal ingroup bias in tasks involving liking/preferences (Baron \& Dunham, 2015; Dunham, Baron, \& Carey, 2011; Elashi \& Mills, 2014; Plötner, Over, Carpenter, \& Tomasello, 2015; Richter, Over, \& Dunham, 2016), helping (Plötner et al., 2015), reputation management (Engelmann, Herrmann, \& Tomasello, 2018; Engelmann, Over, Herrmann, \& Tomasello, 2013), loyalty (Misch, Over, \& Carpenter, 2016, 2018), choosing whom to trust (Elashi \& Mills, 2014; MacDonald, Schug, Chase, \& Barth, 2013), and recalling/interpreting others' actions (Dunham et al., 2011; Dunham \& Emory, 2014; Schug, Shusterman, Barth, \& Patalano, 2013; see Dunham, 2018, and 
Otten, 2016, for recent reviews). These biases are also persistent (Yang \& Dunham, 2019).

Using the minimal group paradigm to explore the roots of group bias (Dunham, 2018) requires that the paradigm truly allows us to probe effects of group assignment. But most minimal group studies are fertile ground for interference from demand characteristics. For example, experimenters commonly assign children to a group based on color, and then have them choose or rate other individuals of the same or a different color. This design could impel participants to choose, or answer favorably about, others wearing the same color they themselves were assigned based on implicit or explicit interpretations of the experimenter's expectations (Durgin et al., 2009; Gerard \& Hoyt, 1974; Orne, 1962).

Could demand characteristics contribute to findings of minimal group bias? Although a few studies have investigated this question, some directly and others indirectly, the relevant evidence is perhaps inconclusive and based mostly on very different methodologies from those typically used in today's work with young children. St. Claire and Turner (1982) used a "prediction" condition that maintained the demand characteristics of the paradigm but removed the assignment of participants to groups, asking participants to predict how someone who was in a group would allocate resources to ingroup and outgroup members. They found no evidence of ingroup bias in this "prediction" condition but did in the "control" condition (a standard minimal group paradigm), suggesting that group membership, not experimental demand, causes bias. Berkowitz (1994) instead assigned all 
participants to minimal groups and, on different trial blocks, asked them to allocate resources to ingroup and outgroup members in a way that confirmed the experimenter's expectations, in a way that went against these expectations, and in whatever way they wanted. Participants generally showed higher levels of ingroup bias when they were responding as they thought they were expected to than when they were responding counter to these perceived expectations, and true ingroup bias scores in the free response trials correlated positively with bias scores in the confirmexpectations trials. This suggested that participants were able to determine that experimenters thought they would prefer their ingroup, and were answering in ways that agreed with these expectations. Thus, these two investigations of demand characteristics in the minimal group paradigm arrived at different conclusions. Gerard and Hoyt (1974) attempted to reduce demand characteristics in their minimal group study, with findings suggesting that there is at least a tendency toward ingroup bias under some circumstances, even when demand characteristics are reduced. A recent child minimal group study also arguably reduced the demand characteristics of the paradigm. Yang and Dunham (2019, Study 2) employed a "minimal" condition in which participants were randomly assigned to a minimal group, but were then switched into the other group. This likely highlighted to participants that group membership was truly meaningless, and that the researcher thought this as well, which might have made them feel less pressure to prefer their ingroup. Ingroup bias did not appear in the "minimal" condition on measures of preference and similarity, but it did in a resource allocation task. This result shows the formation of some kinds 
of minimal group bias in the minimal group paradigm even when demand characteristics are reduced, suggesting that demand characteristics are not the cause of at least certain forms of ingroup bias in this paradigm.

Importantly, potential effects of demand characteristics have not been directly tested in the context of the modern child minimal group paradigm. Even widely supported findings in other areas of experimental psychology have fallen prey to similar complications in recent years (e.g. Durgin et al., 2009; Firestone \& Scholl, 2016). While concerns about demand characteristics may be partially mitigated by recent findings of minimal group bias in implicit measures (e.g. Dunham et al., 2011; Dunham \& Emory, 2014), these are not necessarily immune. Moreover, most minimal group studies use explicit measures.

Here, we aimed to determine whether demand characteristics might contribute to the appearance of bias in the child minimal group paradigm. One approach to exploring the potential role of demand characteristics is to remove them from the paradigm and see if an effect lessens or disappears, thus suggesting that experimental demand created or contributed to the original effect, as in Durgin and colleagues' (2009) classic work on behavioral potential influencing perception. Because removing potential demand cues is very difficult in the minimal group paradigm, we used a different approach: setting up a scenario that should not elicit the effect of interest (Firestone \& Scholl, 2016). We aimed to preserve potential demand characteristics while removing the typically accepted cause of ingroup bias (minimal group membership). To achieve this, we created a novel condition in which 
experimenters introduced a salient color to participants, but did not assign them to groups or mention groups at all. If group membership is the source of minimal ingroup bias, then bias should not emerge in this condition. If, on the other hand, demand characteristics contribute to apparent ingroup preferences in the minimal group paradigm, then bias might emerge in this novel condition.

Our preregistered study included two main between-subjects conditions. The "group" condition used a typical minimal group paradigm, based on Dunham et al. (2011), in which participants were assigned to a green or orange minimal group and wore a green or orange smock. In the novel "no-group" condition, participants were assigned a salient colored object (a green or orange box). In both conditions, participants completed three measures to assess their preferences for photos of children wearing green or orange. These measures determined whether participants liked color-match individuals more than nonmatch individuals, whether they attributed more positive and fewer negative behaviors to color-match individuals, and whether they expected color-match individuals to exhibit more sharing/reciprocity toward them. If participants were biased toward favoring color-match individuals only in the group condition, this would suggest that group membership, not demand characteristics, causes true ingroup bias in this paradigm. However, if color-match bias emerged in both conditions, this would suggest that demand characteristics might contribute to the appearance of bias in minimal group studies.

\section{Method}

\section{Participants}


A total of 165 children participated ( 80 female, 85 male, $M=5.4$ years, $S D=$ 0.57, range 4.5 to 6.7 ). Participants were excluded for failure to complete at least two measures (preregistered grounds for exclusion; $n=1)$, experimenter error $(n=3)$, or parental interference $(n=1)$. The final sample included 160 children (78 female, 82 male, $M=5.4$ years, $S D=0.58$, range 4.5 to 6.7 , with $n=474$-year-olds, $n=855$ year-olds, and $n=28$ 6-year-olds), our preregistered sample size, yielding $>80 \%$ power to detect effects in the small to moderate range for all preregistered analyses (eighty participants per sample needed for within-condition analyses to detect effects of $d=0.3$ with $85 \%$ power or $d=0.4$ with $97 \%$ power; and for between-condition analyses to detect effects of $d=0.4$ with $81 \%$ power). The participant age range was based on the age range used by Dunham et al. (2011). Child participants were recruited from a participant database and at local preschools and a local science center. Testing was completed in a quiet laboratory room on campus $(n=148)$, at the science center $(n=7)$, and in quiet spaces outside preschool classrooms $(n=10)$. The focus here is on the child sample, but we also conducted a supplementary experiment with adults, yielding similar results.

\section{Design}

Participants were randomly assigned to either a "group" or a "no-group" condition ( $n=80$ per condition). In both conditions, participants were randomly assigned to a color (green or orange) prior to the study session. The group condition used a standard minimal group paradigm. In an initial induction phase, group condition participants were placed in a color group (with color predetermined by 
random assignment prior to the session). Children picked a colored wooden "coin" hidden in one of the experimenter's hands, they received a smock of that color to wear, and they were told they were "in the green/orange group." The no-group condition completed a closely matched initial induction phase in which participants were presented with a salient color (again, with color predetermined by random assignment prior to the session). They selected a colored "coin" like participants in the group condition, but for the no-group condition there was no group assignment and the word "group" was never used. Instead, these participants were shown either a green or an orange box that was placed near them for the duration of the study, and they were told they would be "using the green/orange box." Boxes were 6-inch cardboard cubes. The induction phase and accompanying language were matched as closely as possible across conditions. For example, the word "green" (or "orange") was spoken the same number of times in both conditions.

The experimenter informed participants in the group condition that they would be presented with photos of children in the green and orange groups on a laptop in front of them. Participants in the no-group condition were instead told that they would see photos of children wearing green and orange. Participants provided verbal assent to confirm that they would answer some questions about the pictured children. They then completed three measures designed to assess preferences for children wearing green or orange. These measures were identical across conditions, and pre-recorded audio (the same for both conditions) accompanied the tasks whenever possible in order to avoid any condition-based differences in participants' experiences that might 
resulting from the experimenter's knowledge of condition or hypothesis. The emergence of color-match preferences in both main conditions would signify that the introduction of a salient colored object (not group membership per se) is sufficient to induce preferences for people wearing that color.

\section{Measures}

Three measures of group preference were adapted from Dunham et al. (2011): Explicit Attitude (EA), Behavioral Attribution (BA), and Expectations of Reciprocity (ER). In the Explicit Attitude measure, participants saw one photo per trial of a child wearing green or orange and rated how much they liked each child (6 trials: 3 green, 3 orange). A Likert-type scale showing six varied "smiley" faces, ranging from a large smile on the left to a large frown on the right, was used to obtain liking ratings. In each trial of the Behavioral Attribution measure, participants viewed two child photos (one in green, one in orange) presented side by side. They heard a description of a positive behavior (e.g. "Who helped their parents clean the house?") or a negative behavior (e.g. "Who took some money without asking?"), and pointed at the child they thought performed that behavior ( 8 trials: 4 positive, 4 negative). In the Expectations of Reciprocity measure, participants also viewed photos of two children (one in green, one in orange) presented side by side on every trial. For each pair of photos, they heard a description of a behavior involving sharing or reciprocity (e.g. "Which of these kids would definitely share their lunch with you?") and then were asked to choose which of the two children they thought performed that behavior (4 trials). 
There were four possible measure orders (EA/BA/ER, EA/ER/BA, BA/ER/EA, and ER/BA/EA). Explicit Attitude was always first or last to minimize equipment changes during the session, because Behavioral Attribution and Expectations of Reciprocity measures both included pre-recorded audio and headphones were used if ambient noise levels were high. For each of the four measure orders, two unique face photo orders were created such that each participant viewed one of eight possible photo orders. Face photo order was randomly assigned, ensuring random assignment of measure order. Participants were randomly assigned to one of 32 possible combinations of factors ( 2 conditions x 2 colors $\times 4$ measure orders $\mathrm{x} 2$ face photo orders per measure order).

Photos were adapted (by coloring neutral smocks green or orange) from the Child Affective Facial Expression (CAFE) set (LoBue, 2014), developed by LoBue and Thrasher (2015). Photographs of 60 children were used (30 boys, 30 girls; 8 African American, 4 Asian, 12 Caucasian/European American, and 6 Latino children per gender). Each child photo was used in both a green-smock and an orange-smock form (120 child photos total, with 60 unique children). Male participants saw photos of boys and female participants saw photos of girls. All photographs used in the Explicit Attitude measure were of Caucasian/European American children. Photographs used in the Behavioral Attribution and Expectations of Reciprocity measures were of children of different races/ethnicities across trials, but race/ethnicity was always matched within each pair of simultaneously presented photos. Paired photos were also roughly matched by hair color and intensity of positive facial 
expression. There were 30 photos used per testing session, so that each participant saw each distinct same-gender child in the stimulus set exactly one time (and each pictured child could only be wearing one of the two possible colors in a particular session).

Two potentially important differences exist between our stimuli and those used by Dunham et al. (2011). First, in Experiment 2 of Dunham et al. (2011), participants viewed six unique gender-matched child photographs in total, with the same six photographs used throughout all measures, such that the same individuals were viewed multiple times. In the present study, participants were presented with 30 different gender-matched children, each appearing only once. This ensured that participants were making independent judgments about each child, removing the possibility that participants might become more inclined to choose a particular child or to respond in the same way about them if they appeared multiple times, which could potentially lead to a spurious inflation of ingroup bias measures. Second, Dunham et al. (2011) included only European American children in their stimulus set, as is common in minimal group studies when they use child photographs. In our Explicit Attitude measure, all photos depicted Caucasian/European American children, but the photos included children of different races/ethnicities in the Behavioral Attribution and Expectations of Reciprocity measures (across, but not within, trials).

\section{Procedure}


In the induction phase, the experimenter held two coins of the same color behind her back, asking the participant to "pick one of my hands to choose a coin that's orange or green." Following selection, the experimenter said, "You picked the green/orange coin!" This assigned a predetermined (randomly-assigned) color to each participant so that exactly $80 / 160$ participants were assigned each of the two colors. Depending on condition, two smocks or boxes, one of each color, were in the participant's view. In the group condition, the experimenter told the participant they were in the "green/orange group" and gave them a smock of that color to wear. Groups were explicitly labeled. In the no-group condition, the experimenter told the participant they were going to be "using the green/orange box," and placed the appropriate box close to the participant.

The test phase followed with three bias measures (Explicit Attitude, Behavioral Attribution, Expectations of Reciprocity). The order in which measures appear below does not reflect presentation order.

Explicit Attitude. In the Explicit Attitude measure, participants were told they would see photos of children their age and tell how much they liked them. Then the experimenter introduced the Likert-type rating scale. Participants were told they could point to a face to show the experimenter how much they liked each child they saw. Faces were explained from left to right and were verbally labeled as the experimenter pointed to each one ("really like," "like," "kind of like," "kind of don't like," "don't like," and "really don't like"). The participant was asked to indicate the face they would point to if they really didn't like a child, and then the face they would 
point to if they kind of liked a child. If they chose the wrong face, they were shown the correct face and the scale was re-explained. Both confirmation questions were then asked again. If the participant was unsuccessful at this point (after experiencing the scale explanation twice), the experimenter showed the right face and moved on. Thirty-five of the final 160 participants failed to answer both questions correctly after either the first or the second explanation. (Analysis of the subset who were included in Explicit Attitude analyses and successfully answered these questions, $n=124$, yielded the same results as the analysis that included the above 35 participants.) Six test trials followed, each consisting of one photograph of a child (three wearing green, three wearing orange). The experimenter asked the participant how much they liked the child; the participant responded by pointing to a face on the scale. If the participant understood the task, the experimenter did not necessarily repeat the full question on every trial.

Behavioral Attribution. In Behavioral Attribution, the experimenter told the participant they would "listen to the computer," as this measure included pre-recorded audio. If Behavioral Attribution preceded Expectations of Reciprocity for that participant (as in the EA/BA/ER and BA/ER/EA sequences), and if ambient noise levels were high and headphones were to be used for that particular participant, the child chose a pair of headphones to wear. The experimenter wore another pair. Both were plugged into an audio splitter so the experimenter could monitor the audio. If ambient noise levels were low, audio was played directly from the computer speakers. First, audio on an instructional slide was played: participants were informed they 
would see pictures of two children their age, would "hear about something that one of them did," and would point to whichever child they thought performed that behavior. To confirm understanding, the experimenter asked them to explain what they were going to do in the task. If the participant failed to do this, the experimenter read the directions aloud. On each test trial, photographs of two children, one wearing green and the other orange, were shown side by side. The participant heard either a positive behavior or a negative behavior (a subset of behaviors from Dunham et al., 2011) described through pre-recorded audio, and chose the child they thought had done it.

Expectations of Reciprocity. Expectations of Reciprocity (ER) was similar to Behavioral Attribution. The participant was first told that they would "listen to the computer." If Expectations of Reciprocity preceded Behavioral Attribution in a participant's measure order (as in EA/ER/BA and ER/BA/EA sequences), headphones (if needed) were introduced at the start of Expectations of Reciprocity rather than Behavioral Attribution. Pre-recorded instructions told participants they would see pictures of children and point to one of the children to answer the questions they'd hear. The experimenter asked participants to explain what they were going to do in the task, and read the instructions aloud if the participant needed to hear them a second time. Each test trial consisted of the presentation of two side-by-side photos of children, one in orange and one in green. Via pre-recorded audio, the participant listened to questions involving sharing or reciprocity and pointed to select the child they thought would act in that manner. Questions were those used in Dunham et al. (2011). 
Following the three measures, the experimenter took the assigned smock (box) and placed it back next to the non-assigned smock (box). In a brief post-test, the participant was asked why they thought they made the choices that they did, if they recalled the color of the smock (box), and why they thought they had been wearing a smock of that color (why they thought there had been a box of that color on the table). The primary reason for asking these questions was to see if our participants could articulate ideas they had about the study's purpose (Durgin et al., 2009; Orne, 1962). Finally, we recorded whether the participant asked about the purpose of the smock (box) at any point.

\section{Results}

Our preregistered analyses (https://aspredicted.org/4hd8m.pdf) had three main goals. First, we aimed to determine whether participants in the group condition demonstrated color-match bias for each of the three measures (bias favoring photos of individuals in the participant's ingroup, wearing the same color as the participant). Second, we asked whether participants in the no-group condition displayed colormatch bias for each of the three measures (bias favoring photos of individuals wearing the color that matched the assigned box). Third, we asked whether colormatch bias was significantly stronger in the group condition compared to the nogroup condition for each of the three measures. Additionally, exploratory Bayesian analyses were performed in R (R Core Team, 2018), with the "BayesFactor" package 
(Morey \& Rouder, 2018), in order to determine the relative strength of the null versus the alternative for each of the main analyses. ${ }^{1}$

\section{Explicit Attitude Measure}

Data from 1/160 participants were excluded from analyses for noncompletion, leaving 159 participants. Raw data were 6 liking ratings (one per trial, three ratings for photos with smocks matching the participant's color and three ratings of nonmatch photos) made on a six-point scale, ranging from one ("really don't like") to six ("really like"). For each participant, ratings were averaged to yield a single match rating and a single nonmatch rating. Bias scores were calculated by subtracting each participant's mean nonmatch rating from their mean match rating. A positive bias score indicates greater liking for match individuals, a negative score suggests greater liking for nonmatch individuals, and a score of zero signifies no preference. Individual bias scores are plotted in Figure 1 and results are given in Table 1. For the group condition, ratings for color-match photos (ingroup children) were not significantly greater than ratings for nonmatch photos (outgroup children): participants in the group condition did not show evidence of a color-match (ingroup) bias. Bayes factors provide weak support for this null finding $\left(\mathrm{BF}_{01}=2.43\right)$ according to guidelines suggested by Raftery (1995), e.g., Bayes factors of 1-3 provide "weak evidence" while Bayes factors of 3-20 provide "positive evidence" (for full guidelines see Table 6 in Raftery, 1995). For the no-group condition, ratings for color-match photos were not higher than ratings for nonmatch photos: participants in the no-group

\footnotetext{
${ }^{1}$ We thank an anonymous reviewer for suggesting these analyses.
} 
condition also did not display a color-match bias. Bayes factors provide positive support for this null finding $\left(\mathrm{BF}_{01}=14.77\right)$. Bias scores were not significantly greater in the group condition than in the no-group condition. Bayes factors provide weak support for this null finding $\left(\mathrm{BF}_{01}=1.14\right)$.

\section{Behavioral Attribution Measure}

Behavioral Attribution data from 3/160 participants were excluded for noncompletion, leaving a final sample of 157 for these analyses (Table 1; Figure 1). Raw data consisted of participants' choices of color-match or nonmatch individuals from pairs of child photos on eight total trials (four describing positive behaviors, four describing negative behaviors). A bias score (out of eight) was calculated for each participant: the sum of all trials where the participant chose a match face when a positive behavior was described and a nonmatch face when a negative behavior was described. Higher bias scores indicate an expectation that color-match children would engage in positive behaviors and nonmatch children would engage in negative behaviors. A score of four corresponds to chance. In the group condition, bias scores were not significantly greater than chance: participants did not demonstrate a colormatch (ingroup) bias. Bayes factors provide weak support for this null finding $\left(\mathrm{BF}_{01}\right.$ =2.91). No-group condition participants also showed no color-match bias. Bayes factors provide positive support for this null finding $\left(\mathrm{BF}_{01}=9.32\right)$. The group condition bias score was not significantly higher than the no-group score. Bayes factors provide weak support for this null finding $\left(\mathrm{BF}_{01}=2.67\right)$.

\section{Expectations of Reciprocity Measure}


Data from 2/160 participants were excluded for non-completion, leaving 158 participants for these analyses (Table 1; Figure 1). Raw data consisted of participants' choices of photos of either match or nonmatch faces (presented in pairs) on four total trials. A bias score (out of four) was calculated for each participant by summing trials on which match faces were selected. High scores indicate selections of color-match individuals exhibiting sharing or reciprocity. A score of two corresponds to chance. In the group condition, bias scores were significantly greater than chance: participants in the group condition expressed a color-match (ingroup) bias in the Expectations of Reciprocity measure, $d=0.379$. Bayes factors provide strong support for this finding $\left(\mathrm{BF}_{10}=42.08\right)$. In the no-group condition, bias scores were not significantly greater than chance, indicating no color-match bias. Bayes factors provide positive support for this null finding $\left(\mathrm{BF}_{01}=8.07\right)$. Participants in the group condition had significantly higher bias scores than participants in the no-group condition, $d=0.376$. Bayes factors provide positive support for this finding $\left(\mathrm{BF}_{10}=4.34\right)$.

\section{Additional Analyses}

Additional planned analyses explored potential gender differences and effects of color assignment. For Explicit Attitude, group condition bias scores did not differ by sex, $t(78)=0.82, p=0.415$ (two-tailed), nor did no-group condition scores, $t(77)=$ $-0.64, p=0.524$ (two-tailed). The same was true for Behavioral Attribution for group, $t(77)=-0.33, p=0.742$ (two-tailed), and for no-group scores, $t(76)=-1.43, p=0.157$ (two-tailed). The same was true for Expectations of Reciprocity for group, $t(77)=-$ $0.76, p=0.450$ (two-tailed), and for no-group scores, $t(77)=0, p=1.000$ (two- 
tailed). Responses of male and female participants did not differ in any of the three measures in either condition. For Explicit Attitude, children assigned green vs. children assigned orange did not differ in bias scores in the group condition, $t(78)=$ $1.27, p=0.208$ (two-tailed), or the no-group condition, $t(77)=-0.35, p=0.727$ (twotailed). The same was true for Behavioral Attribution for group, $t(77)=1.18, p=$ 0.242 (two-tailed), and no-group, $t(76)=-0.74, p=0.462$ (two-tailed). The same was true for Expectations of Reciprocity for group, $t(77)=-0.46, p=0.647$ (two-tailed), and no-group, $t(77)=-1.46, p=0.148$ (two-tailed). Whether a participant was assigned green or orange did not affect bias on any measure in either condition. Additional planned analyses revealed no correlations between measures, both when responses were examined separately by condition and when they were examined across all participants. Planned analyses also investigated whether participants chose match photos significantly more than chance in the Behavioral Attribution measure, regardless of whether they had heard a positive or negative behavior. The number of Behavioral Attribution trials in which participants selected match photos was not greater than chance in the group or no-group condition.

We also conducted exploratory analyses to test for the presence of colormatch bias in the Explicit Attitude measure with only the subset of participants who had completed the Explicit Attitude task first (and had therefore seen only photos of Caucasian/European American children at that point in the session). These participants (like the full set of participants) did not rate match photos significantly higher than nonmatch photos in either the group or the no-group condition. 
Finally, exploratory linear regression analyses were conducted to assess the relation of age and bias score. ${ }^{2}$ We conducted a linear regression analysis for each measure to determine whether age in months and condition predicted bias scores across all participants, as well as whether there was an interaction of age in months and condition. In the Explicit Attitude measure, there was no main effect of either age $(\beta=0.38, p=0.176)$ or condition $(\beta=0.37, p=0.865)$ on bias score, nor was there an interaction effect $(\beta=-0.01, p=0.972)$. In the Behavioral Attribution measure, there was again no significant main effect of either age $(\beta=0.28, p=0.376)$ or condition $(\beta=-0.28, p=0.910)$ on bias score, nor was there an interaction effect $(\beta=$ $0.08, p=0.854)$. In the Expectations of Reciprocity measure, there was a significant main effect of age $(\beta=0.43, p=0.041)$ but not of condition $(\beta=1.82, p=0.258)$, and there was no interaction of age and condition $(\beta=-0.27, p=0.363)$. Increasing age was related to increased bias scores in the Expectations of Reciprocity measure.

Post-test responses suggested that participants generally did not discern the study's purpose. One participant failed to recall assigned color in the conclusion phase. Of 160 participants in our final sample, only 11 asked about the purpose of the smock (box) at any point. Only a very small proportion of responses suggested children had any sense of the study's purpose.

\section{Supplementary Experiment with Adult Participants}

Adult participants $(N=64,50$ female, 14 male; $n=52$ age range 19-22 years, $M=19.3, S D=1.23 ; n=10$ age range $29-62$ years, $M=46.5, S D=10.76$; two

\footnotetext{
${ }^{2}$ We thank an anonymous reviewer for suggesting these analyses.
} 
additional participants provided no age information) completed the same experiment on campus in exchange for course credit or a small thank-you gift (see adult preregistration https://aspredicted.org/97ny9.pdf). No adult participants were excluded.

Key findings from the supplementary adult sample (Figure 2; Table 2) were very similar to those from the child sample. In the Explicit Attitude and Expectations of Reciprocity analyses, data from all 64 participants were included; in the Behavioral Attribution analyses, data from 1/64 participants were excluded, leaving a total of 63 participants for that measure. In the Explicit Attitude measure, participants did not rate color-match photos more highly than nonmatch photos in either the group condition or the no-group condition. Bayes factors provide weak support for the null finding in the group condition $\left(\mathrm{BF}_{01}=1.68\right)$ and positive support for the null finding in the no-group condition $\left(\mathrm{BF}_{01}=3.93\right)$. Bias scores in the Explicit Attitude measure were not significantly higher in the group condition as compared to the no-group condition. Bayes factors provide weak support for this null finding $\left(\mathrm{BF}_{01}=2.07\right)$. In the Behavioral Attribution measure, participants' bias scores were not significantly above chance in either the group condition or the no-group condition. Bayes factors provide weak support for the null finding in the group condition $\left(\mathrm{BF}_{01}=1.18\right)$ and positive support for the null finding in the no-group condition $\left(\mathrm{BF}_{01}=6.25\right)$. Bias scores in the Behavioral Attribution measure were not significantly greater in the group condition than in the no-group condition. Bayes factors provide weak support for this null finding $\left(\mathrm{BF}_{01}=1.20\right)$. In the Expectations of Reciprocity measure, bias 
scores of participants in the group condition were significantly above chance, $d=$ 0.446. Bayes factors provide positive support for this finding $\left(\mathrm{BF}_{10}=5.56\right)$. Bias scores of participants in the no-group condition were not significantly above chance. Bayes factors provide weak support for this null finding $\left(\mathrm{BF}_{01}=2.59\right)$. Bias scores were not significantly greater in the group condition than in the no-group condition. Bayes factors provide weak support for this null finding $\left(\mathrm{BF}_{01}=1.06\right)$.

\section{Discussion}

There were three main findings; we focus on the child sample that was the main emphasis of this study throughout the discussion. First, in the group condition, ingroup preference emerged in one of the three bias measures only, and second, this preference emerged even though participants evaluated ingroup/outgroup photos that varied in race/ethnicity between trials. We return to these findings below, focusing first on our main question of interest: Could demand characteristics contribute to the appearance of minimal ingroup bias? Addressing this question requires results consistent with ingroup bias in the group condition, but such results were found only in the Expectations of Reciprocity measure. As a result, our ability to address the originally targeted question was somewhat limited. Participants in the no-group condition showed no color-match bias for Expectations of Reciprocity, and bias scores were greater in the group condition than the no-group condition. Thus, colormatch bias was found in the group condition, where group assignment occurred, but not in the no-group condition, where group assignment did not occur but potential 
demand characteristics (introduction of a salient color) were retained. This measure therefore provided no evidence that demand characteristics contribute to ingroup bias here, and our findings are consistent with the idea that minimal ingroup bias, at least in the context of children's expectations about who will share or reciprocate, results from mere membership in a group.

Findings in the adult sample were largely the same as findings in the child sample. Considering adult participants in the group condition, we again found ingroup bias in only the Expectations of Reciprocity measure. Participants in the nogroup condition again did not show a color-match bias in this measure (or in the Explicit Attitude and Behavioral Attribution measures). We did find that bias scores in the Expectations of Reciprocity measure were not significantly greater in the group condition than in the no-group condition for adults, while they were for children. However, the supplementary adult study may have been underpowered to detect a relatively small between-group difference. Overall, findings from the adult study agree with findings from the child study and suggest that in the Expectations of Reciprocity measure, ingroup bias is caused by minimal group assignment and not experimental demand.

Although the child group condition constituted a relatively large, preregistered attempt to replicate established findings of minimal group bias, two measures (Explicit Attitude and Behavioral Attribution) yielded no bias. It is possible that procedural differences between the current study and Dunham et al. (2011), the model for the typical minimal group component of our study, reduced the likelihood that we 
would observe behavior consistent with ingroup bias. In our study, a participant was never presented with the same child photo more than once. In Experiment 2 of Dunham et al. (2011), participants viewed each face multiple times such that their judgments from trial to trial were not independent (see Methods), a design that could have inflated measurements of ingroup bias. But such an explanation would not apply widely to prior reports of minimal group bias.

We also used photos of children of different races/ethnicities in the Behavioral Attribution and Expectations of Reciprocity measures, while Dunham et al. (2011) used photos of only European American children, like most minimal group studies using face photos. We included different races/ethnicities because (1) we did not expect our sample, or their peer groups, to be entirely comprised of European American children, and (2) intergroup bias findings are arguably even more interesting if they emerge in more ecologically valid contexts. We held race/ethnicity constant within face photo pairs, but could across-trial variation in race/ethnicity have reduced children's attention to the color-based minimal group manipulation? We think this is unlikely for two reasons. First, if minimal ingroup bias were "washed out" by across-trial variation, we would expect this to occur broadly. However, though race/ethnicity varied across trials in Expectations of Reciprocity, ingroup bias did emerge in this measure. Second, though race/ethnicity of child photos in Explicit Attitude did not vary (so one might expect ingroup bias to emerge more readily in this task), we found no evidence of bias in this measure, even when we analyzed the subset of participants who completed it first (and had not yet seen any variation in 
race/ethnicity). Altogether, variation in stimulus race/ethnicity is probably not a viable explanation for the absence of minimal group bias in two of three measures.

It could be possible that we did not observe ingroup bias in the Explicit Attitude measure because we used a Likert-type scale to assess participants' liking for color-match and nonmatch photos. Some studies have used forced-choice measures to assess ingroup biases in liking or preference tasks (Baron \& Dunham, 2015; Plötner et al., 2015; Richter et al., 2016; Yang \& Dunham, 2019). Forced-choice measures might be better than scale measures at detecting smaller effects, so it is possible that the inclusion of a forced-choice liking task in our study might have shown an ingroup bias that our Likert-type scale did not detect. Some previous studies have similarly not found ingroup bias in tasks that assess liking with scale measures (Dunham \& Emory, 2014; Schug et al., 2013). However, Dunham et al. (2011) did find ingroup bias using a scale in the same kind of task, and our participants also did not display an ingroup bias in the Behavioral Attribution measure, which was a forced-choice task.

More likely is that ingroup bias emerged only in Expectations of Reciprocity because this task differs from the others in some way. Expectations of Reciprocity (but not the other two measures) may require participants to think about how others would treat ingroup vs. outgroup members. Explicit Attitude and Behavioral Attribution, on the other hand, involve no reasoning about others' conception of group relations. Relatedly, Dunham (2018) theorized that there are two main types of ingroup bias, caused by different factors. He proposed that people might have "evaluative" ingroup biases, viewing the ingroup in positive ways because we view 
ourselves in positive ways, and "coalitional" ingroup biases, which form because we have certain beliefs about how group members should interact with each other. Of the measures in our study, Explicit Attitude and Behavioral Attribution would be classified as measures of evaluative kinds of bias, and Expectations of Reciprocity is likely a measure of the coalitional form. Given that we only found ingroup bias in the Expectations of Reciprocity task, perhaps certain coalitional forms of bias are stronger than evaluative forms. Findings from Yang and Dunham (2019) are consistent with this idea: after decreasing the meaningfulness of minimal groups, these researchers also found ingroup bias in a coalitional measure (resource allocation) but not in an evaluative measure (preference), which again suggests that coalitional biases may be stronger.

Other evidence is also consistent with the idea that ingroup bias in Explicit Attitude and Behavioral Attribution tasks is weak not just in the present study, but more generally. For example, of the Explicit Attitude, Behavioral Attribution, and Expectations of Reciprocity measures, the largest effect size was for Expectations of Reciprocity in a previous study (Dunham et al., 2011, Exp. 2). There seems to be no clear consensus on whether ingroup bias forms reliably in Explicit Attitude and Behavioral Attribution measures. Though several studies have found evidence of ingroup bias in these tasks (Dunham et al., 2011; Elashi \& Mills, 2014; Richter et al., 2016 for Explicit Attitudes; Wu \& Gao, 2018), others have not (Dunham \& Emory, 2014; Misch et al., 2016; Richter et al., 2016 for Behavioral Attribution; Schug et al., 
2013), and still others have done so only under certain circumstances (Baron \& Dunham, 2015; Plötner et al., 2015).

In the minimal group paradigm, differences between to-be-evaluated individuals are often minimized so findings of bias can be attributed solely to group membership. Thus, few minimal group studies have participants evaluate photos of people who vary in race/ethnicity. The finding of ingroup bias in our Expectations of Reciprocity measure, where race/ethnicity varied across trials, is therefore unusual. Guassi Moreira, Van Bavel, and Telzer (2016) included this kind of stimulus variation, and their 8- to 16-year-old participants displayed a greater liking for photos of people in their ingroup than for photos of those in the outgroup. Even though Guassi Moreira et al. (2016) found bias in a different measure, focused on older children, and did not create strictly minimal groups, this is notably similar to the present work. In another study, Rhodes, Leslie, Saunders, Dunham, and Cimpian (2018) assigned 4.5- to 6-year-olds to a "Gorp" group, making an outgroup of the "Zarpies" (a group including individuals varying in age, gender, and race/ethnicity). After hearing a neutral-valence story about "Zarpies," participants did show biases favoring their "Gorp" ingroup. Though race/ethnicity was not heavily discussed in this study, it did assess children's biases for ingroup and outgroup members that varied along these dimensions, as in the present study. Our work provides converging evidence supporting the use of diversified stimuli in minimal group research, both to create more inclusive studies and to determine whether ingroup biases emerge in more scenarios than might previously have been thought. 
Overall, our findings are consistent with the generally accepted view that mere membership in a group, not experimental demand, leads participants to exhibit minimal ingroup preferences. We interpret this result conservatively, though, because the main hypothesis about the potential role of demand characteristics could only be tested for one of the three measures. These findings also may raise questions about the replicability of minimal ingroup preferences, given the lack of ingroup bias in Explicit Attitude and Behavioral Attribution measures in this large-scale, preregistered context. However, another way to interpret this result is as a contribution toward establishing a baseline of theoretically useful null results. As Dunham (2018) points out, "failures to find a particular effect in the MGP are as informative as successes" (p. 786). This is because we need reliable evidence regarding effects that do vs. do not arise in this paradigm in order to use the paradigm as a tool for separating biases following from mere membership from those arising from specific instances of cultural or social learning (Dunham, 2018). Additional evidence regarding successful or unsuccessful replications from other preregistered, well-powered studies will be needed to settle these questions.

A potential limitation of this study is power, considering the possibility that minimal group effects are real but quite small. The present work was well powered to detect small to moderate minimal group preferences, with sample size chosen with respect to both the effect size we wished to detect and practical constraints on the number of child participants that could realistically participate. Although the sample size for this study is larger than those reported in much published work on the MGP, 
it is possible that smaller effects do exist that would not have been detected in our sample of 160 participants ( 80 per condition). Taken together, the reported Bayes factors might reasonably be interpreted as consistent with this idea: for the two measures that did not produce findings of ingroup bias, there was positive evidence for the null in the no-group conditions but only weak evidence for the null in the group conditions. Future research aimed at detecting smaller effects, e.g. in the $d=$ 0.2 range, would need considerably larger samples. Another potential limitation is the idea that the no-group condition in our study might actually include a less direct form of group assignment, rather than no group assignment at all as intended. It could be argued that simply being presented with an object of one of two colors is a form of group assignment, and if this is the case, then perhaps our no-group condition is more of a "weaker-group" condition. However, we think we have achieved a reasonable no-group manipulation; if simply noting that a colored object is present is a form of group assignment, then many things could be interpreted as a form of group assignment.

In future work, it would be ideal for researchers to test the possibility of influence from demand characteristics in a similar manner using additional measures of minimal ingroup bias (see Dunham, 2018; Otten, 2016, for reviews). For example, some studies have found evidence of ingroup bias in more implicit measures (e.g. Dunham et al., 2011; Dunham \& Emory, 2014). In Dunham et al. (2011), minimal ingroup bias was strongest in an implicit attitude measure. By applying the present study design to new contexts, including implicit bias measures and a wider range of 
explicit measures, future research could provide strong tests of the potential influence of demand characteristics in the minimal group paradigm. 


\section{References}

Baron, A. S., \& Dunham, Y. (2015). Representing 'us' and 'them': Building blocks of intergroup cognition. Journal of Cognition and Development, 16, 780-801.

Berkowitz, N. H. (1994). Evidence that subjects' expectancies confound intergroup bias in Tajfel's minimal group paradigm. Personality and Social Psychology Bulletin, 20, 184-195.

Dunham, Y. (2018). Mere membership. Trends in Cognitive Sciences, 22, 780-793.

Dunham, Y., Baron, A. S., \& Carey, S. (2011). Consequences of “minimal” group affiliations in children. Child Development, 82, 793-811.

Dunham, Y., \& Emory, J. (2014). Of affect and ambiguity: The emergence of preference for arbitrary ingroups. Journal of Social Issues, 70, 81-98.

Durgin, F. H., Baird, J. A., Greenburg, M., Russell, R., Shaughnessy, K., \& Waymouth, S. (2009). Who is being deceived? The experimental demands of wearing a backpack. Psychonomic Bulletin \& Review, 16, 964-969.

Elashi, F. B., \& Mills, C. M. (2014). Do children trust based on group membership or prior accuracy? The role of novel group membership in children's trust decisions. Journal of Experimental Child Psychology, 128, 88-104.

Engelmann, J. M., Herrmann, E., \& Tomasello, M. (2018). Concern for group reputation increases prosociality in young children. Psychological Science, 29, 181-190. 
Engelmann, J. M., Over, H., Herrmann, E., \& Tomasello, M. (2013). Young children care more about their reputation with ingroup members and potential reciprocators. Developmental Science, 16, 952-958.

Firestone, C., \& Scholl, B. J. (2016). Cognition does not affect perception: Evaluating the evidence for "top-down" effects. Behavioral and Brain Sciences, 39.

Gerard, H. B., \& Hoyt, M. F. (1974). Distinctiveness of social categorization and attitude toward ingroup members. Journal of Personality and Social Psychology, 29, 836-842.

Guassi Moreira, J. F., Van Bavel, J. J., \& Telzer, E. H. (2016). The neural development of 'us and them'. Social Cognitive and Affective Neuroscience, 12, 184-196.

LoBue, V. (2014). The Child Affective Facial Expression (CAFE) set. Databrary. http://doi.org/10.17910/B7301K

LoBue, V., \& Thrasher, C. (2015). The Child Affective Facial Expression (CAFE) set: Validity and reliability from untrained adults. Frontiers in Emotion Science, 5, 1532.

MacDonald, K., Schug, M., Chase, E., \& Barth, H. (2013). My people, right or wrong? Minimal group membership disrupts preschoolers' selective trust. Cognitive Development, 28, 247-259.

Misch, A., Over, H., \& Carpenter, M. (2016). I won't tell: Young children show loyalty to their group by keeping group secrets. Journal of Experimental Child Psychology, 142, 96-106. 
Misch, A., Over, H., \& Carpenter, M. (2018). The whistleblower's dilemma in young children: When loyalty trumps other moral concerns. Frontiers in Psychology, 9,250 .

Morey, R. D., \& Rouder, J. N. (2018). BayesFactor: Computation of Bayes factors for common designs (Version 0.9.12-4.2) [R package]. Available from https://CRAN.R-project.org/package=BayesFactor

Mullen, B., Brown, R., \& Smith, C. (1992). Ingroup bias as a function of salience, relevance, and status: An integration. European Journal of Social Psychology, $22,103-122$.

Orne, M. T. (1962). On the social psychology of the psychological experiment: With particular reference to demand characteristics and their implications. American Psychologist, 17, 776-783.

Otten, S. (2016). The Minimal Group Paradigm and its maximal impact in research on social categorization. Current Opinion in Psychology, 11, 85-89.

Plötner, M., Over, H., Carpenter, M., \& Tomasello, M. (2015). The effects of collaboration and minimal-group membership on children's prosocial behavior, liking, affiliation, and trust. Journal of Experimental Child Psychology, 139, 161-173.

R Core Team (2018). R: A language and environment for statistical computing (Version 3.4.4) [Software]. Vienna, Austria: R Foundation for Statistical Computing. Available from https://www.R-project.org/ 
Raftery, A. E. (1995). Bayesian model selection in social research. Sociological Methodology, 25, 111-163.

Rhodes, M., Leslie, S. J., Saunders, K., Dunham, Y., \& Cimpian, A. (2018). How does social essentialism affect the development of inter-group relations? Developmental Science, 21, e12509.

Richter, N., Over, H., \& Dunham, Y. (2016). The effects of minimal group membership on young preschoolers' social preferences, estimates of similarity, and behavioral attribution. Collabra, 2, 8 .

Schug, M. G., Shusterman, A., Barth, H., \& Patalano, A. L. (2013). Minimal-group membership influences children's responses to novel experience with group members. Developmental Science, 16, 47-55.

St. Claire, L., \& Turner, J. C. (1982). The role of demand characteristics in the social categorization paradigm. European Journal of Social Psychology, 12, 307314.

Tajfel, H., Billig, M. G., Bundy, R. P., \& Flament, C. (1971). Social categorization and intergroup behaviour. European Journal of Social Psychology, 1, 149178.

Wu, Z., \& Gao, X. (2018). Preschoolers' group bias in punishing selfishness in the Ultimatum Game. Journal of Experimental Child Psychology, 166, 280-292.

Yang, X., \& Dunham, Y. (2019). Minimal but meaningful: Probing the limits of randomly assigned social identities. Journal of Experimental Child Psychology, 185, 19-34. 
Table 1

Children: Results of Preregistered Analyses

\begin{tabular}{llll}
\hline \multicolumn{1}{c}{ Analysis } & EA & Measures & \\
& $M_{\text {match }}=4.06(1.14)$ & & ER \\
Group & {$[3.81,4.32]$} & & \\
Condition & $M_{\text {nonmatch }}=3.88(1.19)$ & $M=4.18(1.54)$ & $M=2.41(1.07)$ \\
& {$[3.61,4.14]$} & {$[3.83,4.52]$} & {$[2.17,2.64]$} \\
& $t(79)=1.17$ & $t(78)=1.02$ & $t(78)=3.37$ \\
& $p=0.123$ & $p=0.155$ & $p<0.001$ \\
& & & \\
No-Group & $M_{\text {match }}=4.04(1.21)$ & & \\
Condition & {$[3.77,4.31]$} & $M=3.96(1.69)$ & $M=2.00(1.09)$ \\
& $M_{\text {nonmatch }}=4.19(1.10)$ & {$[3.58,4.34]$} & {$[1.76,2.24]$} \\
& $t(78)=-0.95$ & $t(77)=-0.20$ & $t(78)=0$ \\
& $p=0.173$ & $p=0.420$ & $p=0.5$ \\
Group vs. No- & $M_{\text {no-group }}=-0.15(1.43)$ & $t(155)=0.84$ & $t(156)=2.36$ \\
Group & {$[-0.47,0.17]$} & $p=0.201$ & $p=0.010$ \\
& $t(157)=1.49$ & & \\
& $p=0.069$ & & \\
\hline
\end{tabular}

Note. Standard deviations are reported in parentheses, $95 \%$ confidence intervals of the means in brackets. All $p$-values are one-tailed. All significant $p$-values remain so following Bonferroni correction for three comparisons $(\alpha=0.0167)$. In the Explicit Attitude measure, means reported for group and no-group condition analyses are mean rating scores for color-match and nonmatch photos; means reported for the group vs. no-group analysis of this measure are bias scores (in the EA measure, bias score $=$ mean match rating - mean nonmatch rating, and was calculated for both conditions so that a between-conditions comparison could be performed). In the Behavioral Attribution and Expectations of Reciprocity measures, bias scores were used for all three main analyses: the means reported in the group and no-group condition cells for BA and ER are mean bias scores and are the values that were compared in the group vs. no-group analyses for these two measures. 
Table 2

Adults: Results of Preregistered Analyses

\begin{tabular}{|c|c|c|c|}
\hline \multirow[b]{2}{*}{ Analysis } & \multicolumn{3}{|c|}{ Measures } \\
\hline & EA & $\mathrm{BA}$ & ER \\
\hline $\begin{array}{l}\text { Group } \\
\text { Condition }\end{array}$ & $\begin{array}{l}M_{\text {match }}=4.94(0.93) \\
{[4.60,5.27]} \\
M_{\text {nonmatch }}=4.70(0.99) \\
{[4.34,5.05]} \\
t(31)=1.15 \\
p=0.129\end{array}$ & $\begin{array}{l}M=4.41(1.62) \\
{[3.82,4.99]} \\
t(31)=1.42 \\
p=0.083\end{array}$ & $\begin{array}{l}M=2.44(0.98) \\
{[2.08,2.79]} \\
t(31)=2.52 \\
p=0.009\end{array}$ \\
\hline $\begin{array}{l}\text { No-Group } \\
\text { Condition }\end{array}$ & $\begin{array}{l}M_{\text {match }}=4.67(0.87) \\
{[4.35,4.98]} \\
M_{\text {nonmatch }}=4.61(0.96) \\
{[4.27,4.96]} \\
t(31)=0.36 \\
p=0.361\end{array}$ & $\begin{array}{l}M=3.94(1.44) \\
{[3.41,4.46]} \\
t(30)=-0.25 \\
p=0.402\end{array}$ & $\begin{array}{l}M=2.13(0.91) \\
{[1.80,2.45]} \\
t(31)=0.78 \\
p=0.221\end{array}$ \\
\hline $\begin{array}{l}\text { Group vs. No- } \\
\text { Group }\end{array}$ & $\begin{array}{l}M_{\text {group }}=0.24(1.18) \\
{[-0.18,0.66]} \\
M_{\text {no-group }}=0.05(0.82) \\
{[-0.24,0.35]} \\
t(62)=0.74 \\
p=0.231\end{array}$ & $\begin{array}{l}t(61)=1.22 \\
p=0.114\end{array}$ & $\begin{array}{l}t(62)=1.32 \\
p=0.096\end{array}$ \\
\hline
\end{tabular}

Note. Standard deviations are reported in parentheses, 95\% confidence intervals of the means in brackets. All $p$-values are one-tailed. The significant $p$-value remains so following Bonferroni correction for three comparisons $(\alpha=0.0167)$. In the Explicit Attitude measure, means reported for group and no-group condition analyses are mean rating scores for color-match and nonmatch photos; means reported for the group vs. no-group analysis of this measure are bias scores (in the EA measure, bias score = mean match rating - mean nonmatch rating, and was calculated for both conditions so that a betweenconditions comparison could be performed). In the Behavioral Attribution and Expectations of Reciprocity measures, bias scores were used for all three main analyses: the means reported in the group and no-group condition cells for BA and ER are mean bias scores and are the values that were compared in the group vs. no-group analyses for these two measures. 

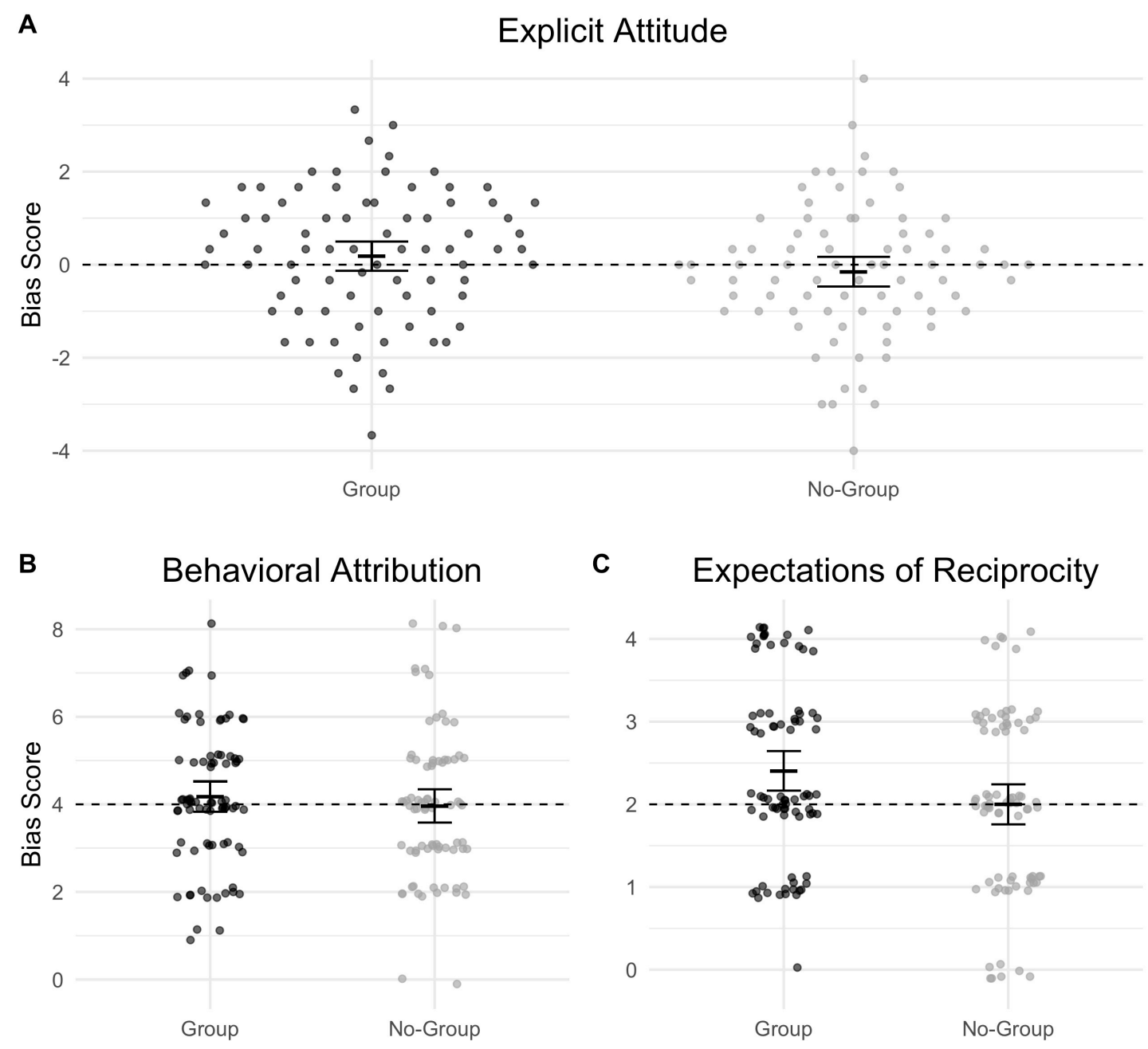

Figure 1. Individual child bias scores by condition in the Explicit Attitude (A), Behavioral Attribution (B), and Expectations of Reciprocity (C) measures. In each plot, the dashed horizontal line indicates chance. Scores above chance suggest a greater preference for match children, and scores below chance suggest a greater preference for nonmatch children. For each condition, mean bias scores and $95 \%$ confidence intervals are shown (see Table 1). The position of points along the horizontal axis (within condition) is arbitrary. In the BA and ER measures, bias scores are integer values; in plots B and C, points are jittered such that these scores generally appear slightly above or below their true values. 
A

Explicit Attitude
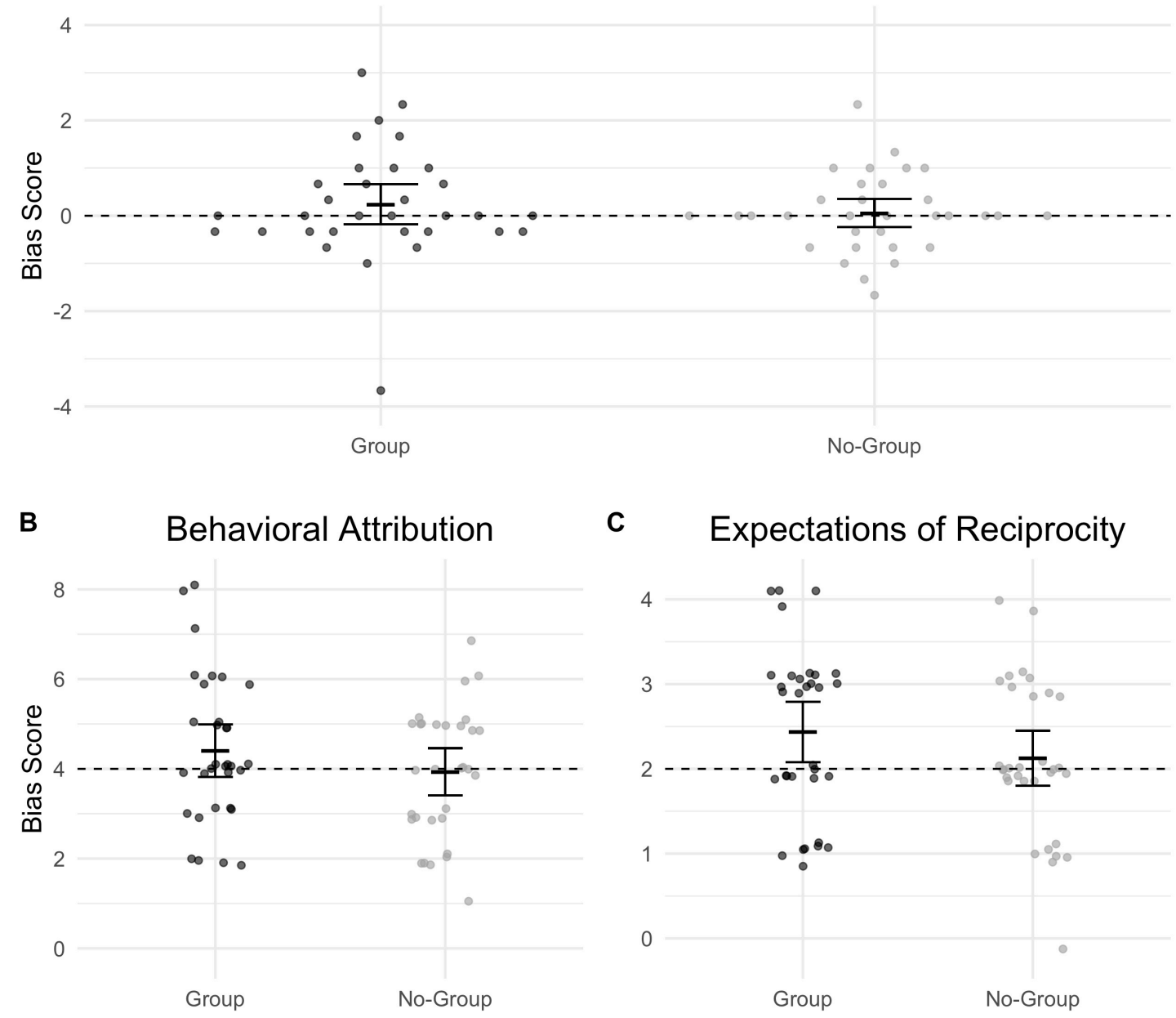

Figure 2. Individual adult bias scores by condition in the Explicit Attitude (A), Behavioral Attribution (B), and Expectations of Reciprocity (C) measures. In each plot, the dashed horizontal line indicates chance. Scores above chance suggest a greater preference for match children, and scores below chance suggest a greater preference for nonmatch children. For each condition, mean bias scores and $95 \%$ confidence intervals are shown (see Table $\mathrm{C} 1$ ). The position of points along the horizontal axis (within condition) is arbitrary. In the BA and ER measures, bias scores are integer values; in plots B and C, points are jittered such that these scores generally appear slightly above or below their true values. 